\title{
Utilizing the CGC/saturation approach for soft scattering at high energies
}

\author{
E. Gotsman ${ }^{1, a}$ \\ ${ }^{1}$ School of Physics and Astronomy, Tel Aviv University, Tel Aviv, 69978, Israel
}

\begin{abstract}
We propose a consistent approach to describe soft interactions at LHC and higher energies based on the BFKL Pomeron and CGC/saturation. The Green function of the Pomeron is calculated in the framework of CGC/saturation approach. Having eight parameters we obtained a good description of the experimental data at high energies ( $W \geq 0.546 \mathrm{TeV})$.
\end{abstract}

\section{Introduction}

Strong interactions at high energy are considered as a typical example of processes that occur at long distances, where the unknown confinement of quark and gluons plays a crucial role making all theoretical efforts to treat these processes, fruitless. As a result the description of these soft processes, is the arena of high energy phenomenology based on Pomeron calculus [1].

To overcome this, we suggest an approach which attempts to describe soft interactions by adopting well established aspects of QCD in the saturation region [2], and extending these to the region of soft interactions (low $Q^{2}$ ). Our basic assumption, is that the BFKL Pomeron [3], at high energies, describes both hard and soft processes.

\section{Theoretical Input}

In this section we briefly review our model which succesfully describes elastic, diffractive [4, 5], inclusive cross sections [6] and long range rapidity correlations [7] The main ingredient of our model is the BFKL [3] Pomeron Green function, that we obtained using a CGC/saturation approach [8]. We determined this function from the solution of the non-linear Balitsky-Kovchegov equation [9], using the MPSI approximation [10] to sum enhanced diagrams, shown in Fig. 1-a. It has the following form:

$$
\begin{aligned}
& G^{\text {dressed }}(T)=\quad a^{2}(1-\exp (-T))+2 a(1-a) \frac{T}{1+T}+(1-a)^{2} G(T) \\
& \text { with } \quad G(T)=1-\frac{1}{T} \exp \left(\frac{1}{T}\right) \Gamma_{0}\left(\frac{1}{T}\right)
\end{aligned}
$$

\footnotetext{
ae-mail: gotsman@post.tau.ac.il
} 


$$
T(s, b)==\phi_{0} S(b, m) e^{0.63 \lambda \ln \left(s / s_{0}\right)} \text { with } S(b, m)=\frac{m^{2}}{2 \pi} e^{-m b}
$$

See [5] for the role of the variables.

We use a two channel model [5], based on the Good-Walker [11] formalism, which allows us to calculate the diffractive production in the region of small masses.

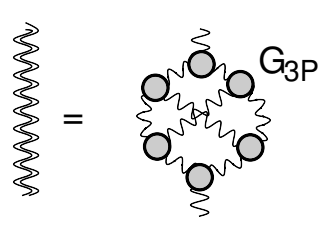

a)

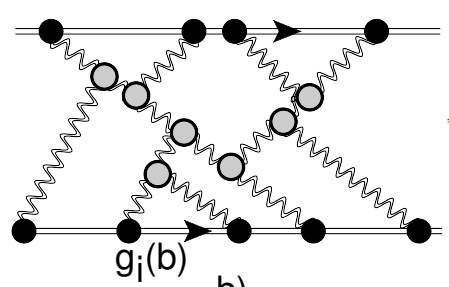

b)

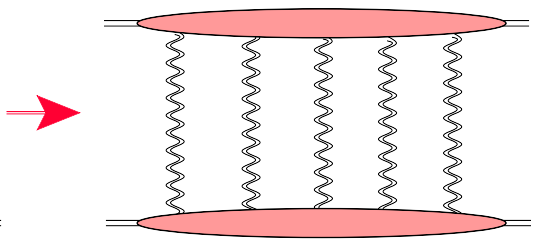

c)

Figure 1. Fig. 1-a shows the set of the diagrams in the BFKL Pomeron calculus that produce the resulting (dressed) Green function of the Pomeron in the framework of high energy QCD. In Fig. 1-b the net diagrams which include the interaction of the BFKL Pomerons with colliding hadrons are shown. The sum of the diagrams reduces to Fig. 1-c after integration over positions of $G_{3 P}$ in rapidity.

In the eikonal approach [5] we parametrize the arbitrary functions $\Omega_{i k}(s, b)$ (opacity) in the form

$$
\Omega_{i k}(s, b)=\int d^{2} b^{\prime} d^{2} b^{\prime \prime} g_{i}\left(m_{i}, b^{\prime}\right) g_{k}\left(m_{k} b^{\prime \prime}\right) G^{\text {dressed }}\left(T\left(Y, \vec{b}-\vec{b}^{\prime}-\vec{b}^{\prime \prime}\right)\right),
$$

The summation of the 'net' diagram, is then given by the following simplified expression (see Ref. [12] for details)

$$
\Omega(s, b)_{i, k}=\int d^{2} b^{\prime} \frac{g_{i}\left(\vec{b}^{\prime}\right) g_{k}\left(\vec{b}-\vec{b}^{\prime}\right) \widetilde{G}^{\text {dressed }}(T)}{1+1.29 \widetilde{G}^{\text {dressed }}(T)\left[g_{i}\left(\vec{b}^{\prime}\right)+g_{k}\left(\vec{b}-\vec{b}^{\prime}\right)\right]},
$$

where

$$
\widetilde{G}^{\text {dressed }}(T)=\int d^{2} b G^{\text {dressed }}(T(Y, b)) .
$$

The coefficient 1.29 results from the extraction of the value of $G_{3 \boldsymbol{P}}$ in the CGC/saturation approach.

Note that our choice of impact parameter dependence i.e. $\mathrm{S}(\mathrm{b}) \propto \exp (-m b)$ leads to the correct Froissart limit at large $\mathrm{b}$, and at large momentum transfer $\left(Q_{T}\right)$ it decreases as a power of $Q_{T}$, as required in perturbative QCD.

\section{Diffraction production in the region of large mass}

In this section we also include, in the process of diffraction production, the mechanism of production that originates from the dressed Pomeron, and has been discussed in section 2. 
For single diffraction the large mass contribution can be written

$$
\begin{aligned}
& \sigma_{s d}^{\text {large mass }}=2 \int d^{2} b \\
& \left\{\alpha^{6} A_{1 ; 1,1}^{s d} e^{-2 \Omega_{1,1}^{D}(Y ; b)}+\alpha^{2} \beta^{4} A_{1 ; 2,2}^{s d} e^{-2 \Omega_{1,2}^{D}(Y ; b)}+2 \alpha^{4} \beta^{2} A_{1 ; 1,2}^{s d} e^{-\left(\Omega_{1,1}^{D}(Y ; b)+\Omega_{1,2}^{D}(Y ; b)\right)}\right. \\
& \left.+\beta^{2} \alpha^{4} A_{2 ; 1,1}^{s d} e^{-2 \Omega_{1,2}^{D}(Y ; b)}+2 \beta^{4} \alpha^{2} A_{2 ; 1,2}^{s d} e^{-\left(\Omega_{1,2}^{D}(Y ; b)+\Omega_{2,2}^{D}(Y ; b)\right)}+\beta^{6} A_{2 ; 2,2}^{s d} e^{-2 \Omega_{2,2}(Y ; b)}\right\} .
\end{aligned}
$$

where

$$
\begin{aligned}
& \Omega_{i, k}^{D}(Y ; b)=\int d^{2} b^{\prime} \frac{g_{i}\left(\vec{b}^{\prime}\right) g_{k}\left(\vec{b}-\vec{b}^{\prime}\right) \bar{G}^{\text {dressed }}(T)}{\left(1+1.29 \bar{G}^{\text {dressed }}(T)\left[g_{i}\left(\vec{b}^{\prime}\right)+g_{k}\left(\vec{b}-\vec{b}^{\prime}\right)\right]\right)^{2}} \\
& A_{i ; k, l}^{s d}\left(Y, Y_{\text {max }}, Y_{\text {min }} ; b\right)= \\
& \int d^{2} b^{\prime} \sigma_{\text {diff }}\left(Y, Y_{\text {max }}, Y_{\min }, 1 / m\right) d^{2} b^{\prime} g_{i} g_{k} g_{l} S_{\boldsymbol{P}}\left(b^{\prime}, m_{i}\right) S_{\boldsymbol{P}}\left(\vec{b}-\vec{b}^{\prime}, m_{k}\right) S_{\boldsymbol{P}}\left(\vec{b}-\vec{b}^{\prime}, m_{l}\right),
\end{aligned}
$$

where $Y=\ln \left(s / s_{0}\right), Y_{\max }=\ln \left(M_{\max }^{2} / s_{0}\right)$ and $Y_{\min }=\ln \left(M_{\min }^{2} / s_{0}\right) . M_{\max }$ and $M_{\min }$ are the largest and smallest mass produced in the diffractive processes. Eq. (3.6) has simple physical meaning: each term is the product of probability to produce a mass diffractively from the dressed Pomeron (term $\exp \left(-\Omega_{i, k}\right)$ ), and the probability of the process of single diffraction, from the dressed Pomeron $\left(A_{i ; k, l}\right)$.

For the double diffraction production at large mass we have

$$
\begin{gathered}
\sigma_{d d}^{\text {large mass }}=\int d^{2} b\left\{\alpha^{4} A_{1,1}^{d d} e^{-2 \Omega_{1,1}^{D}(Y ; b)}+2 \alpha^{2} \beta^{2} A_{1,2}^{d d} e^{-2 \Omega_{1,2}^{D}(Y ; b)}+\beta^{4} A_{2,2}^{d d} e^{-2 \Omega_{2,2}^{D}(Y ; b)}\right\} \\
A_{i, k}^{d d}=\int d^{2} b g_{i} g_{k} S_{D D}^{i, k}(b) \sigma_{d d}(Y) \quad \text { where } S_{D D}^{i, k}(b)=\int d^{2} b^{\prime} S_{p}\left(b^{\prime}, m_{i}\right) S_{p}\left(\vec{b}-\vec{b}^{\prime}, m_{k}\right),
\end{gathered}
$$

The expressions for $\sigma_{\text {diff }}\left(Y, Y_{\max }, Y_{\min }, r\right)$ and $\sigma_{d d}$ are given in [5]

\section{Fitting to Experimental data}

There are eight phenomenological parameters, which need to be determined by fitting to the experimental data. $\phi_{0}, \lambda$ and $m, g_{i}$ and $m_{i}(\mathrm{i}=1,2)$, as well as $\beta$. We determine these parameters by fitting to the experimental data on total, inelastic and elastic cross sections, single and double diffractive production cross sections, and the slope of the forward elastic differential cross section. The value of the minimal energy for data that we use is $W=0.546 \mathrm{TeV}$, as starting from this energy the CGC/saturation approach, is able to describe the data on inclusive production in proton-proton collisions (see Ref. [13]).

The values of the fitted parameters are given in Table I. Our results are shown in Fig. 2, and we obtain very good agreement with the experimental data, except for $\sigma_{d d}$, where our results are below the experimental points. 


\begin{tabular}{|l|l|l|l|l|l|l|l|l|}
\hline model & $\lambda$ & $\phi_{0}$ & $g_{1}\left(\mathrm{GeV}^{-1}\right)$ & $g_{2}\left(\mathrm{GeV}^{-1}\right)$ & $m(\mathrm{GeV})$ & $m_{1}(\mathrm{GeV})$ & $m_{2}(\mathrm{GeV})$ & $\beta$ \\
\hline 2 channel & 0.38 & 0.0019 & 110.2 & 11.2 & 5.25 & 0.92 & 1.9 & 0.58 \\
\hline
\end{tabular}

Table 1. Fitted parameters of the model.

\section{Conclusions}

We have shown that a consistent model, based on the BFKL Pomeron and the CGC/saturation approach, can be built. We have demonstrated that this model successfully describes data for high energy hadron scattering usually classified as "soft" i.e elastic and diffractive cross sections [4, 5], inclusive cross sections [6] and long range correlations [7]. In addition, we hope that this paper provides credence to the arguments, that the matching with long distance physics, (where the confinement of quarks and gluons is essential), can be reached within the CGC/saturation approach, this, without requiring that the soft Pomeron should appear (as a Regge pole).

\section{Acknowledgements}

This work was done in collaboration with Evengy Levin and Uri Maor. I would like to thank my colleagues for a fruitful and enjoyable joint effort.

\section{References}

[1] P.D.B. Collins, An introduction to Regge theory and high energy physics (Cambridge University Press, 1977).

[2] Yuri V Kovchegov and Eugene Levin, Quantum Choromodynamics at High Energies", (Cambridge Monographs on Particle Physics, Nuclear Physics and Cosmology, Cambridge University Press, 2012) .

[3] E. A. Kuraev, L. N. Lipatov, and F. S. Fadin, Sov. Phys. JETP 45, 199 (1977); Ya. Ya. Balitsky and L. N. Lipatov, Sov. J. Nucl. Phys. 28, 22 (1978).

[4] E. Gotsman, E. Levin and U. Maor, Eur. Phys. J. C 75, 18 (2015).

[5] E. Gotsman, E. Levin and U. Maor, Eur. Phys. J. C 75, 179 (2015).

[6] E. Gotsman, E. Levin and U. Maor, Phys. Lett. B741, 154 (2015).

[7] E. Gotsman, E. Levin and U. Maor, Eur. Phys. J. C 75, 518 (2015).

[8] E. Levin, JHEP 1311, 039 (2013).

[9] I. Balitsky, [arXiv:hep-ph/9509348]; Phys. Rev. D60, 014020 (1999) Y. V. Kovchegov, Phys. Rev. D60, 034008 (1999), [arXiv:hep-ph/9901281].

[10] A. H. Mueller and B. Patel, Nucl. Phys. B425, 471 (1994). A. H. Mueller and G. P. Salam, Nucl. Phys. B475, 293 (1996). G. P. Salam, Nucl. Phys. B461, 512 (1996); E. Iancu and A. H. Mueller, Nucl. Phys. A730, 460 (2004)

[11] M. L. Good and W. D. Walker, Phys. Rev. 1201857 (1960).

[12] E. Gotsman, E. Levin and U. Maor, Int.J.Mod.Phys. A30, 98 (2015). arXiv:1403.4531 [hep-ph].

[13] E. Levin and A. H. Rezaeian, Phys. Rev. D 83, 114001 (2011). [arXiv:1102.2385 [hep-ph]], AIP Conf. Proc. 1350 (2011) 243 [arXiv:1011.3591 [hep-ph]], Phys. Rev. D 82, 054003 (2010).

[14] J. Beringer et al. (Particle Data Group), Phys. Rev. D86, 010001 (2012) 
[15] F. Ferro [TOTEM Collaboration], AIP Conf. Proc. 1350, 172 (2011); G. Antchev et al. [TOTEM Collaboration], Europhys. Lett. 96, 21002 (2011), Europhys. Lett. 96, 21002 (2011), Europhys. Lett. 96, 21002 (2011), 95, 41001 (2011)

[16] M. G. Poghosyan, J. Phys. G G 38, 124044 (2011) ALICE Collaboration, J. Phys. G G 38, 124044 (2011) ALICE Collaboration, arXiv:0911.5430 [hep-ex]. 


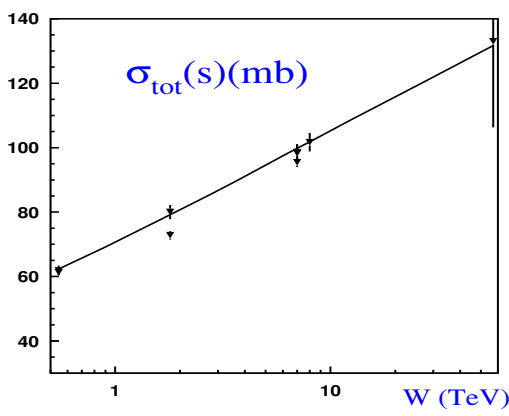

Fig. 2-a

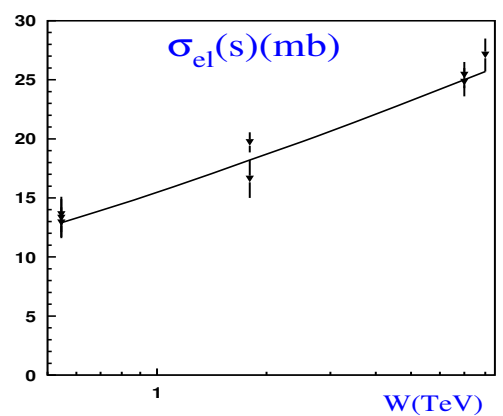

Fig. 2-c

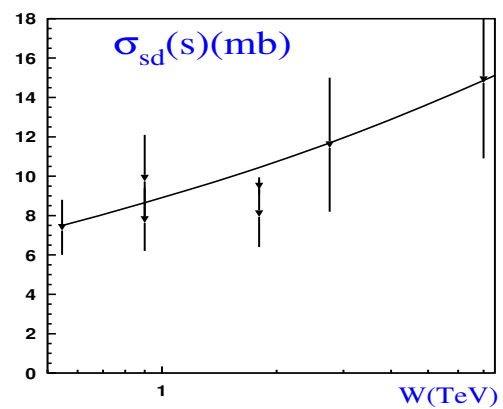

Fig. 2-e

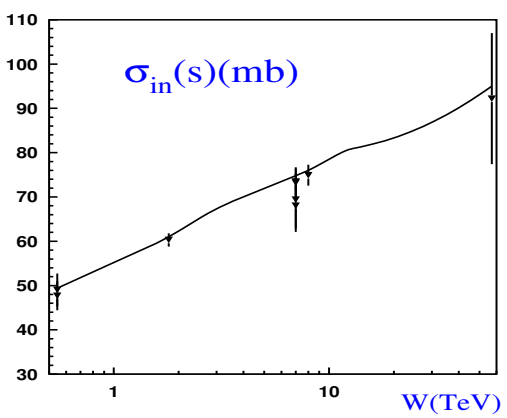

Fig. 2-b

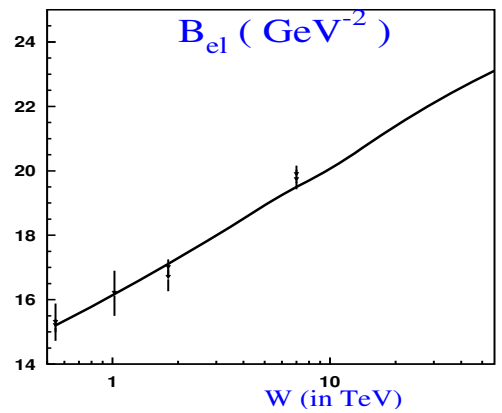

Fig. 2-d

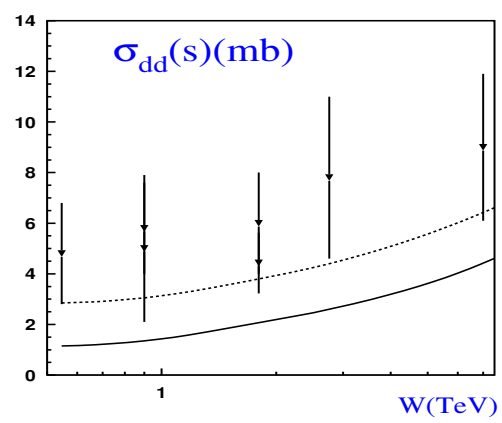

Fig. 2-f

Figure 2. Comparison with the experimental data: the energy behaviour of the total (Fig. 2-a), inelastic (Fig. 2b), elastic cross sections (Fig. 2-c), as well as the elastic slope ( $B_{e l}$,Fig. 2-d) and single diffraction (Fig. 2-e) and double diffraction (Fig. 2-f) cross sections. The solid lines show our present fit. The data has been taken from Ref. [14] for energies less than the LHC energy. At the LHC energy for total and elastic cross section we use TOTEM data [15] and for single and double diffraction cross sections are taken from Ref. [16]. The dotted line in (Fig. 2-f) which is obtained assuming factorization, is discussed in [5]. 